Result. Math. 31 (1997) 300-321

Results in Mathematics

0378-6218/97/040300-22 \$1.50+0.20/0

(C) Birkhäuser Verlag, Basel, 1997

\title{
Smooth Stable Planes
}

\author{
Richard Bödi
}

\begin{abstract}
This paper deals with smooth stable planes which generalize the notion of differentiable (affine or projective) planes [7]. It is intended to be the first one of a series of papers on smooth incidence geometry based on the Habilitationsschrift of the author. It contains the basic definitions and results which are needed to build up a foundation for a systematic study of smooth planes. We define smooth stable planes, and we prove that point rows and line pencils are closed submanifolds of the point set and line set, respectively (Theorem (1.6)). Moreover, the flag space is a closed submanifold of the product manifold $P \times \mathcal{L}$ (Theorem (1.14)), and the smooth structure on the set $P$ of points and on the set $\mathcal{L}$ of lines is uniquely determined by the smooth structure of one single line pencil. In the second section it is shown that for any point $p \in P$ the tangent space $\mathrm{T}_{p} P$ carries the structure of a locally compact affine translation plane $\mathcal{A}_{p}$, see Theorem (2.5). Dually, we prove in Section 3 that for any line $L \in \mathcal{L}$ the tangent space $\mathrm{T}_{L} \mathcal{L}$ together with the set $\delta_{L}=\left\{\mathrm{T}_{L} \mathcal{L}_{p} \mid p \in L\right\}$ gives rise to some shear plane. It turned out that the translation planes $\mathcal{A}_{p}$ are one of the most important tools in the investigation of smooth incidence geometries. The linearization theorems (3.9), (3.11), and (4.4) can be viewed as the main results of this paper. In the closing section we investigate some homogeneity properties of smooth projective planes.
\end{abstract}

AMS-Subject Classification: 51H25, 51A40

Keywords: stable plane, smooth, differentiable incidence geometry, tangent translation plane.

\section{Introduction}

The first characterizations of plane geometry date back more than 2300 years, the most famous text book on this subject being that of Euclid. The axioms of Euclid essentially remained unchanged until M. Pasch published his "Vorlesungen über neuere Geometrie" in 1882. The axioms he used for describing Euclidean geometry introduced the concept of an order. In this way he was able to incorporate continuity properties of the Euclidean plane. The axioms of Pasch reappeared in David Hilbert's book "Grundlagen der Geometrie", published in 1899, and they became well-known to mathematicians. In order to extend the axioms of Pasch and Hilbert to other geometries such as the affine plane over the complex numbers, the concept of an order has to be replaced by a more general idea, namely by that of topology. In 1932 Kolmogoroff was the first who combined projective spaces with topological structures. In the mid-fifties, Skornyakov (1954) and Freudenthal (1957) published two papers on topological planes, and it was Helmut Salzmann who started the systematic investigation of topological plane geometries in 1955. The programme that emanated from his studies is the classification of homogeneous compact connected projective 
planes, where homogeneity is most often measured by the dimensions of their collineation groups. The progress that Salzmann and his school achieved within the last four decades is documented in the recent book Compact Projective Planes [36] that appeared in 1995. Although containing almost 700 pages, only part of the theory developed by now has found its way into this book. This shows the amount of knowledge that has been obtained in this mathematical field, not to mention various other types of topological incidence geometries (see the articles of Steinke and Grundhöfer-Löwen in [13]).

In every classical projective plane $\mathcal{P}$, i.e. the projective plane defined over the real, complex, quaternion, or octonion numbers (which we abbreviate by $\mathbb{R}, \mathbb{C}, \mathbb{H}$, and $\mathbb{O}$, respectively), the geometric operations are not only continuous but even differentiable. Already in 1955 Hellmuth Kneser suggested to study non-Desarguesian smooth planes, but until now only a few isolated papers dealt with differentiable incidence structures. Considering smooth geometries, we have to specify the degree of differentiability to be required. If we regard smooth manifolds as being complex differentiable, then it turns out that the only smooth projective plane is the projective plane over the complex numbers. This was proved by S. Breitsprecher [6] and L. Kramer [25], see also [36], 75.1. Thus we have to use weaker notions of smoothness. The case of real analyticity was also tackled by Breitsprecher. For two-dimensional projective planes he proved in [6] that such a plane $\mathcal{P}$ is isomorphic to the real projective plane (which, of course, is analytic) if $\mathcal{P}$ can be embedded analytically into the complex projective plane $\mathcal{P}_{2} \mathbb{C}$. Up to the present day it is unknown whether this additional embedding assumption is necessary or not. In fact, it seems that the real analytic case is too hard to handle by now. Hence we will use the words smooth and $\mathrm{C}^{\infty}$-differentiable synonymously.

Next we are going to give an overview of the existing literature on differentiable incidence structures. We have mentioned already the pioneer of smooth geometry, S. Breitsprecher, who wrote three papers in the late 60's, [6], [7], [8], on this subject. In 1972, D. Betten published a paper [2] on differentiable two-dimensional projective planes, in which he answered a question of Kneser by showing that the proper Moulton planes cannot be turned into smooth projective planes. After a period of 14 years where nothing was published about smooth incidence geometry, H. Hähl and T. Grundhöfer wrote two papers, [16] and [15], on spherical fibrations which lead to smooth translation planes defined over division algebras. The main result of these two papers is that every smooth translation plane defined over some division algebra is isomorphic to one of the classical projective planes $\mathcal{P}$. J. Otte generalized this result in 1992 to arbitrary translation planes, see [33] and [34]. It is important to note that an analoguous result is false for smooth affine translation planes: Otte constructed a large variety of non-classical examples of smooth affine translation planes and of smooth projective non-translation planes. The most recent results on smooth projective planes are due to L. Kramer [24]. He showed that the point space (and the line space) of a smooth projective plane is homeomorphic to the point space 
of the classical projective plane of the same dimension. For compact connected projective planes in general this is still an open question.

Since smoothness is a local property, it is convenient to adapt the underlying incidence geometry to that local situation. This leads to the concept of a stable plane which is already a well-known object of topological geometry. Stable planes originate from Salzmann's paper Topological planes [35] and have been studied systematically by R. Löwen and by M. Stroppel, see for example [28], [30], [39], and [40].

This manuscript is intended to be the first one of a series of papers on smooth incidence geometry based on the Habilitationsschrift of the author. It contains most of the basic definitions and results which are needed to build up a foundation for a systematic study of smooth planes. It turned out that the tangent translation planes are one of the most important tools in this investigation, see Theorem (3.5). The linearization theorems (3.9), (3.11), and (4.4) can be viewed as the main results of this paper. The author likes to thank $\mathrm{P}$. Taylor for his $\mathrm{T}_{\mathrm{E}} \mathrm{X}$ macro package that easily allows to typeset commutative diagrams. Last but not least I want to thank the referee of this paper for the many valuable remarks and suggestions he made.

\section{Definitions and basic results}

(2.1) Definition. A linear space is a triple $(P, \mathcal{L}, \mathcal{F})$ of sets $P, \mathcal{L}$ and $\mathcal{F}$, where $P$ denotes the set of points, $\mathcal{L}$ is the set of lines and $\mathcal{F} \subseteq P \times \mathcal{L}$ is the set of flags, such that for every pair of distinct points $p, q$ there is exactly one joining line $L \in \mathcal{L}$, i.e. $(p, L),(q, L) \in \mathcal{F}$. If $(p, L) \in \mathcal{F}$, we shall say that $p$ and $L$ are incident, or that $p$ lies on $L$, or that $L$ passes through $p$.

An equivalent formulation of this axiom is that the join map $\vee: P \times P \backslash \operatorname{diag}_{P} \rightarrow \mathcal{L}$ which assigns to each pair of distinct points its joining line is well-defined. Dually, one can speak of the intersection map $\wedge$. Note that in a linear space two lines may not intersect. Hence, for a linear space, the operation $\wedge$ need not be defined on the whole set $\mathcal{L} \times \mathcal{L} \backslash \operatorname{diag}_{\mathcal{L}}$. It is convenient to identify each line $L$ of a linear space with the set of points that are incident with $L$, i.e. we identify $L$ with the set $\{p \in P \mid(p, L) \in \mathcal{F}\}$. If we look at a line this way, we will frequently call it a point row. Similarly, a pencil $\mathcal{L}_{p}$ of lines is the set of all lines that are incident with a given point $p$. A triangle in a linear space $\mathcal{S}$ is a set of three (pairwise distinct) points which do not lie on a common line. A quadrangle is a set of four points such that any three of them form a triangle.

(2.2) Definition. A stable plane $\mathcal{S}$ is a linear space $(P, \mathcal{L}, \mathcal{F})$ which satisfies the following three axioms: 
(S1) There are Hausdorff topologies on both $P$ and $\mathcal{L}$ that are neither discrete nor anti-discrete such that the join map $\vee$ and the intersection map $\wedge$ are continuous. Moreover, the domain $\mathcal{O}_{\wedge}$ of the intersection map is an open subset of $\mathcal{L} \times \mathcal{L}$.

(S2) The topology on $P$ is locally compact and has positive finite covering dimension.

(S3) $\mathcal{S}$ contains a quadrangle.

Note that a projective space of dimension $\geq 3$ over a locally compact skewfield is not a stable plane, because in this case the domain $\mathcal{O}_{\wedge}$ of the intersection map is not open. The following theorem summarizes those properties of stable planes which we will need later. Proofs can be found in Löwen [28] and [30].

(2.3) Theorem. Let $\mathcal{S}=(P, \mathcal{L}, \mathcal{F})$ be a stable plane and let $p \in P, L \in \mathcal{L}$.

a) The point row $L$ is a closed subset of the set $P$ of points, the line pencil $\mathcal{L}_{p}$ is compact, and the flag space $\mathcal{F}$ is a closed subset of $P \times \mathcal{L}$.

b) The set $\mathcal{L}$ of lines and the line pencils $\mathcal{L}_{p}$ are connected.

c) $\operatorname{dim} L=\operatorname{dim} \mathcal{L}_{p}=l=2^{k}$ with $k=0,1,2,3$, and $\operatorname{dim} P=\operatorname{dim} \mathcal{L}=2 l, \operatorname{dim} \mathcal{F}=3 l$, where dim denotes the topological covering dimension. The stable plane $\mathcal{S}$ is said to be $2 l$-dimensional.

d) $\operatorname{dim} U=\operatorname{dim} X$ for every open subset $U$ of $X \in\left\{L, \mathcal{L}_{p}, P, \mathcal{L}, \mathcal{F}\right\}$.

(2.4) Definition. A smooth stable plane $\mathcal{S}$ is a stable plane $(P, \mathcal{L}, \mathcal{F})$ such that $P$ and $\mathcal{L}$ are smooth manifolds and such that the join and intersection maps are smooth on their (respective) domains. If, in addition, $(P, \mathcal{L}, \mathcal{F})$ is a projective plane, then $\mathcal{S}$ is called a smooth projective plane.

Throughout this paper, let $\mathcal{S}=(P, \mathcal{L}, \mathcal{F})$ denote a smooth stable plane of dimension $2 l$ and let $\mathcal{O}_{\wedge}$ be the open domain of the intersection map $\wedge$. Note that in contrast to Breitsprecher [7] we do not require the point rows and the line pencils of $\mathcal{S}$ to be closed submanifolds of $P$ and $\mathcal{L}$, respectively. As we will see below, this follows already from the definition of a smooth stable plane. When speaking of a submanifold, we always mean a smoothly embedded manifold. A map $f: X \rightarrow Y$, where $X$ is a subset of $Y$, is called a retraction onto $A \subseteq Y$, if and only if $f(X)=A$ and $f \circ f=f$. The following criterion for a subset of a smooth manifold to be a smoothly embedded submanifold has turned out to be very useful.

(2.5) Lemma. Let $N$ be a subset of a smooth manifold $M$ such that for every point $x \in N$ there exists a neighborhood base $\mathcal{U}(x)$ of $x$ in $N$ consisting of open neighborhoods of finite covering dimension $n=\operatorname{dim} N$. The set $N$ is a submanifold of $M$ (i.e., the inclusion map $N \hookrightarrow M$ is an embedding), if for every $x \in N$ and every neighborhood $V$ of $x$ in $M$ there exists an open subset $U$ of $x$ in $V$ and a smooth retraction $f: U \rightarrow M$ onto $N \cap U$. 
Proof. The proof of [10], 5.13, shows that $N$ is a local submanifold of $M$, i.e. for every $x \in N$ there is a neighborhood $U$ of $x$ in $M$ such that $N \cap U$ is a submanifold of $M$, see also Hirsch [17], p. 20, Ex. 2. Thus it only remains to show that all these local submanifolds are of uniform dimension. Since, by hypothesis, the covering dimension of $N \cap U$ is $n$, we infer that $N \cap U$ is an $n$-dimensional submanifold, which proves the lemma.

(2.6) Theorem. Every point row $L$ is a closed submanifold of $P$ and every line pencil $\mathcal{L}_{p}$ is a compact submanifold of $\mathcal{L}$.

Proof. Fix a line $L \in \mathcal{L}$ and some point $p \in P \backslash L$. By axiom (S1) of a stable plane, the map $f: U \rightarrow P: x \mapsto(x \vee p) \wedge L$ is defined on some open subset $U$ of $P$ which contains the point row $L$. Clearly we have $f \circ f=f$ and thus $L=f(U)$ is a submanifold of $P$ by (2.5) and (2.3), part d). By Theorem (2.3), part a), the submanifold $L$ is closed in $P$. For the dual statement, let $L \in \mathcal{L}_{p}$ and choose some line $K \in \mathcal{L} \backslash \mathcal{L}_{p}$ which intersects $L$. Consider the smooth map $g: \mathcal{U} \rightarrow \mathcal{L}_{p}: M \mapsto(M \wedge K) \vee p$ which is defined on some open neighborhood $\mathcal{U}$ of $L$ in $\mathcal{L}$. Again, we have $g \circ g=g$, and by (2.5) and (2.3), the line pencil $\mathcal{L}_{p}$ is a compact submanifold of $\mathcal{L}$.

As an easy consequence, we get the following simple but nevertheless important result on perspectivities.

(2.7) Corollary. For $L \in \mathcal{L}$ and $p \in P \backslash L$ the perspectivity $\eta_{p, L}: L \rightarrow \mathcal{L}_{p}: x \mapsto x \vee p$ is a diffeomorphism between $L$ and an open subset of $\mathcal{L}_{p}$, and $\eta_{L, p}: \mathcal{U} \rightarrow L: X \mapsto X \wedge L$ is the inverse map of $\eta_{p, L}$.

Proof. The map $\eta_{p, L}: L \rightarrow \eta_{p, L}(L)$ is a smooth bijection defined on the smooth manifold $L$ whose inverse map is given by some restriction of $\eta_{L, p}: \mathcal{U} \rightarrow L: X \mapsto X \wedge L$ which is smooth as well. By (S1), the domain $\mathcal{U}$ is open and contains the image $\eta_{p, L}(L)$. Since $\eta_{p, L}(L)=\mathcal{L}_{p} \cap \mathcal{U}$, we infer that $\eta_{p, L}(L)$ is an open subset of $\mathcal{L}_{p}$.

It turns out that the smooth structures on the set $\mathcal{L}$ of lines and on the set $P$ of points are uniquely determined by the smooth structures of all line pencils and the geometric operations. In order to prove this, we need two preparatory lemmas. For $p \in P$ we put $\vee_{p}: P \backslash\{p\} \rightarrow \mathcal{L}_{p}: x \mapsto x \vee p$ and dually we set $\wedge_{L}: \mathcal{O}_{L} \rightarrow L: K \mapsto K \wedge L$ for some line $L \in \mathcal{L}$, where $\mathcal{O}_{L} \subset \mathcal{L}$ is the (open) domain of $\wedge_{L}$.

(2.8) Lemma. Let $p, q \in P$ be distinct points, set $L:=p \vee q$ and $\mathcal{L}^{*}:=\mathcal{L}_{p} \backslash\{L\} \times \mathcal{L}_{q} \backslash\{L\}$. Then the map $\chi_{p, q}: P \backslash L \rightarrow \mathcal{L}^{*}: x \mapsto\left(\vee_{p}(x), \vee_{q}(x)\right)$ is a diffeomorphism onto the open subset $X:=\mathcal{L}^{*} \cap \mathcal{O}_{\wedge}$ of $\mathcal{L}^{*}$.

Proof. The set $\mathcal{L}^{*}$ is a submanifold of $\mathcal{L} \times \mathcal{L}$ by Corollary (2.6). By definition of a smooth stable plane, the map $\chi_{p, q}$ is smooth. Since every pair of distinct points determines exactly 
one line, the map $\chi_{p, q}$ is an injection. By definition of the set $\mathcal{O}_{\wedge}$, the image of $\chi_{p, q}$ is just $X$. Moreover, its inverse map is given by the restriction of the intersection map $\wedge$ to $X$ which is smooth, too.

We will also formulate the dual statement of the previous lemma. However, we omit the proof, since it is analogous to the one above.

(2.9) Lemma. Let $K, L \in \mathcal{L}$ be distinct lines, let $p \in K \backslash L, q \in L \backslash K$, and set $M=p \vee q$. Then there is an open neighborhood $\mathcal{U}_{M}$ of $M$ in $\mathcal{L}$ such that the map $\nu_{K, L}: \mathcal{U}_{M} \rightarrow K \times L: X \mapsto\left(\wedge_{K}(X), \wedge_{L}(X)\right)$ is a diffeomorphism onto some open subset of $K \times L$.

(2.10) Corollary. The smooth structures of the set $P$ of points and the set $\mathcal{L}$ of lines is uniquely determined by the smooth structure of one single line pencil $\mathcal{L}_{o}$ and by the geometric operations of $\mathcal{S}$.

Proof. Using Corollary (2.7) we can transfer the given smooth structure on the line pencil $\mathcal{L}_{o}$ to any other line pencil of $\mathcal{S}$. Set $K:=o \vee u, L:=o \vee v$, and $M:=u \vee v$. Since the point rows $K, L$ and $M$ are closed subsets of $P$, the set $\{P \backslash K, P \backslash L, P \backslash M\}$ is an open covering of $P$, and the smooth structures of its members are determined by the smooth structures of the line pencils $\mathcal{L}_{o}, \mathcal{L}_{u}$, and $\mathcal{L}_{v}$ by Lemma (2.8). Thus, the same is true for the smooth structure on $P$. Let $N \in \mathcal{L}$. We may assume that the point $u$ does not lie on $M$. Then the smooth structure on $N$ is given by the map $\vee_{u}: N \rightarrow \mathcal{L}_{u}: n \mapsto u \vee n$, see Corollary (2.7). Thus, the smooth structure of a line in $\delta$ is determined by one of the given line pencils and by the perspectivity $\vee_{u}$. By Lemma (2.9), this implies that the smooth structure on $\mathcal{L}$ only depends on the smooth structure of $\mathcal{L}_{o}$, because the family $\left\{\mathcal{U}_{M} \mid M \in \mathcal{L}\right\}$ is an open covering of $\mathcal{L}$.

A more detailed result on the link between the smooth structures of the set of points and the set of lines which we will prove next, yields that two point rows with a common point $p$ intersect transversally in $p$. Moreover, statement (ii) will play an important rôle in Section 3.

(2.11) Lemma. Let $L_{1}, L_{2}$ be two lines that intersect in some point $p$, and let $q_{i} \in L_{i} \backslash\{p\}$. Then there exist open neighborhoods $U$ of $p$ in $P$ and $\mathcal{U}$ of $L_{1}$ in $\mathcal{L}$ such that

(i) the $\operatorname{map} \varphi: U \rightarrow U_{1} \times U_{2}: x \mapsto\left(\left(x \vee q_{2}\right) \wedge L_{1},\left(x \vee q_{1}\right) \wedge L_{2}\right)$, where $U_{i}:=U \cap L_{i}$, is a diffeomorphism of $U$ onto $U_{1} \times U_{2}$,

(ii) $U \cap L$ is diffeomorphic to $\mathbb{R}^{l}$ for every line $L \in \mathcal{U}$,

(iii) $\rho_{L}: U_{1} \rightarrow U \cap L: x \mapsto\left(x \vee q_{2}\right) \wedge L$ is a diffeomorphism for every line $L \in \mathcal{U}$.

Proof. By (S1), there are neighborhoods $\mathcal{V}_{i}$ of $L_{i}$ in $\mathcal{L}_{q_{i}}$ such that $\mathcal{V}_{1} \times \mathcal{V}_{2} \subseteq \mathcal{O}_{\wedge}$. By Lemma (2.8), the set $\mathcal{V}_{1} \times \mathcal{V}_{2}$ is mapped onto some open neighborhood $U \subseteq P$ of $p$ by 
the diffeomorphism $\chi_{q_{1}, q_{2}}^{-1}$. The map $\varphi$ as defined in the Lemma is well-defined (because of $\left.\mathcal{V}_{1} \times \mathcal{V}_{2} \subseteq \mathcal{O}_{\wedge}\right)$ and smooth on $U$. In order to show that $\varphi$ maps $U$ onto $U_{1} \times U_{2}$, we only have to check that $U_{i}=\left(U \vee q_{3-i}\right) \wedge L_{i}$ holds for $i=1,2$. The inclusion " $\subseteq$ " is trivial, since $x=\left(x \vee q_{3-i}\right) \wedge L_{i}$ for $x \in U_{i}$. Conversely, if we have $x=\left(y \vee q_{3-i}\right) \wedge L_{i}$ for some $y \in U$, then $y \vee q_{3-i} \in \mathcal{V}_{3-i}$. Thus, we infer that $x \in \chi_{q_{1}, q_{2}}^{-1}\left(\mathcal{V}_{1} \times \mathcal{V}_{2}\right)=U$, and hence $x \in U \cap L_{i}=U_{i}$. Since the smooth map

$$
U_{1} \times U_{2} \rightarrow U:\left(u_{1}, u_{2}\right) \mapsto\left(u_{1} \vee q_{2}\right) \wedge\left(u_{2} \vee q_{1}\right)
$$

is the inverse mapping of $\varphi$, this shows that $\varphi$ is a diffeomorphism.

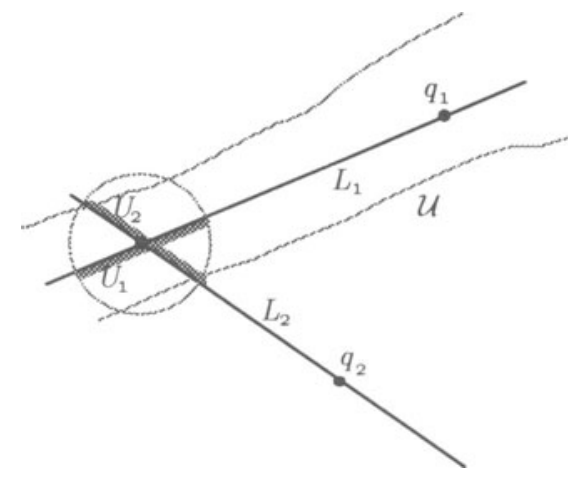

In order to prove statements (ii) and (iii), we choose $\mathcal{V}_{2}$ to be diffeomorphic to $\mathbb{R}^{l}$, which is possible because $\mathcal{L}_{p}$ is an $l$-dimensional manifold by Theorem (2.6). By (i) and Corollary (2.7), the neighborhood $\mathcal{V}_{2}$ is mapped onto $U_{1}$ by the diffeomorphism $K \mapsto K \wedge L_{1}$. Thus, the intersection $U_{1}=L_{1} \cap U$ is diffeomorphic to $\mathbb{R}^{l}$. For any $L \in \mathcal{V}_{1}$, the projectivity $\rho_{L}: U_{1} \rightarrow L: x \mapsto\left(x \vee q_{2}\right) \wedge L$ is well-defined and maps $U_{1}$ onto some open subset $U_{L}$ of $L$. Recall that $\vee_{q_{2}}^{-1}\left(\mathcal{V}_{2}\right)$ is the set of all those points that lie on some line of $\mathcal{V}_{2}$. Since $\vee_{q_{2}}^{-1}\left(V_{2}\right) \cap L_{1} \subseteq U$ and

$$
\vee_{q_{2}}^{-1}\left(\mathcal{V}_{2}\right) \cap L=\rho_{L}\left(U_{1}\right)=\left\{\left(x \vee q_{2}\right) \wedge L \mid x \in U_{1}\right\}
$$

the continuity of the intersection map $\wedge$ gives us some neighborhood $\mathcal{U}^{\prime} \subseteq \mathcal{L}$ of $L_{1}$ such that $\vee_{q_{2}}^{-1}\left(\mathcal{V}_{2}\right) \cap L \subseteq U$ holds for every line $L \in \mathcal{U}^{\prime}$. Setting $\mathcal{U}:=\mathcal{U}^{\prime} \cap \mathcal{V}_{1}$, we conclude that

$$
\mathbb{R}^{l} \cong \rho_{L}\left(U_{1}\right)=\vee_{q_{2}}^{-1}\left(\mathcal{V}_{2}\right) \cap L \subseteq U \cap L
$$

for any line $L \in \mathcal{U}$. By definition of $U$ we have

$$
U=\chi_{q_{1}, q_{2}}^{-1}\left(\mathcal{V}_{1} \times \mathcal{V}_{2}\right)=\vee_{q_{1}}^{-1}\left(\mathcal{V}_{1}\right) \cap \vee_{q_{2}}^{-1}\left(\mathcal{V}_{2}\right) \subseteq \vee_{q_{2}}^{-1}\left(\mathcal{V}_{2}\right),
$$

and hence, relation (*) is in fact an equality. This proves (ii) and (iii). 
In Section 4 we will also need the dual of Lemma (2.11). For convenience we formulate the dual statement and sketch a proof.

(2.12) Lemma. Let $p_{1}, p_{2} \in P$ be two points and set $L=p_{1} \vee p_{2}$. Then there exist open neighborhoods $\mathcal{U}$ of $L$ in $\mathcal{L}$ and $U$ of $p_{1}$ in $P$, and there exist lines $K_{i} \in \mathcal{L}_{p_{i}}$ such that

(i) the map $\psi: \mathcal{U} \rightarrow \mathcal{U}_{1} \times \mathcal{U}_{2}: X \mapsto\left(\left(X \wedge K_{2}\right) \vee p_{1},\left(X \wedge K_{1}\right) \vee p_{2}\right)$, where $\mathcal{U}_{i}:=\mathcal{U} \cap \mathcal{L}_{p_{i}}$, is a diffeomorphism of $\mathcal{U}_{\text {onto }} \mathfrak{U}_{1} \times \mathcal{U}_{2}$,

(ii) $\mathfrak{U} \cap \mathcal{L}_{p}$ is diffeomorphic to $\mathbb{R}^{l}$ for every point $p \in U$,

(iii) $\rho_{p}: \mathcal{U}_{1} \rightarrow \mathcal{U} \cap \mathcal{L}_{p}: X \mapsto\left(X \wedge K_{2}\right) \vee p$ is a diffeomorphism for every point $p \in P$.

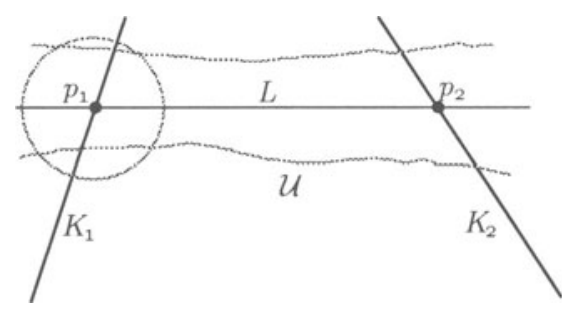

Sketch of proof. Choose lines $K_{i} \in \mathcal{L}_{p_{i}} \backslash\{L\}$. By Lemma (2.9), there are disjoint open neighborhoods $V_{i}$ of $p_{i}$ in $K_{i}$ such that the product $V_{1} \times V_{2}$ is mapped onto some open neighborhood $\mathcal{U} \subseteq \mathcal{L}$ of $L$ by the diffeomorphism $\nu_{K_{1}, K_{2}}^{-1}$. The map $\psi$ defined in the lemma is well-defined by the very definition of $\mathcal{U}$ and is smooth on $\mathcal{U}$. Now we can argue as in the proof of Lemma (2.11) in order to show that $\varphi$ is a diffeomorphism. This proves (i). Now choose $V_{2}$ to be diffeomorphic to $\mathbb{R}^{l}$ by using Lemma (2.6). By (i) and Corollary (2.7), the projectivity $x \mapsto x \vee p_{1}: V_{2} \rightarrow \mathcal{U}_{1}$ is a diffeomorphism, whence $\mathcal{U}_{1} \cong \mathbb{R}^{l}$. Choose an open neighborhood $U \subseteq P$ of $p_{1}$ which is disjoint to $V_{2}$. Then, for each point $p \in U$ the projectivity $\rho_{p}: \mathcal{U}_{1} \rightarrow \mathcal{L}_{p}: X \mapsto\left(X \wedge K_{2}\right) \vee p$ is defined. Since the join map $\vee$ is continuous, we may select $U$ in such a way that $\wedge_{K_{2}}^{-1}\left(V_{2}\right) \cap \mathcal{L}_{p} \subseteq \mathcal{U}$ holds for every $p \in U$. Now we proceed as in Lemma (2.11) in order to prove (ii) and (iii).

(2.13) Corollary. If two lines of $\mathcal{S}$ intersect, they intersect transversally, i.e. if $K, L \in \mathcal{L}$ with $p=K \wedge L$, then there is a coordinate neighborhood $(U, h)$ at $p$ such that $h(U \cap K)=$ $\mathbb{R}^{l} \times\{0\}$ and $h(U \cap L)=\{0\} \times \mathbb{R}^{l}$. Dually, any two line pencils intersect transversally in $\mathcal{L}$.

(2.14) Theorem. The flag space $\mathcal{F}$ of a smooth stable plane $\mathcal{S}$ is a closed submanifold of the product manifold $P \times \mathcal{L}$. 
Proof. Let $(p, L) \in \mathcal{F}$ and select some point $o \in P \backslash L$. By (S1), there are open neighborhoods $U$ of $p$ and $\mathcal{V}$ of $L$ such that $\{x \vee o \mid x \in U\} \times \mathcal{V} \subseteq \mathcal{O}_{\wedge}$. Then the map

$$
f: U \times \mathcal{V} \rightarrow \mathcal{F}:(q, K) \mapsto((q \vee o) \wedge K, K)
$$

is well-defined and smooth. Moreover, we have $f \circ f=f$, since $(q \vee o) \wedge K=q$ if $(q, K) \in \mathcal{F} \cap(U \times \mathcal{V})$. Thus, by Lemma (2.5) and Theorem (2.3), part d), the flag space $\mathcal{F}$ is a submanifold of $P \times \mathcal{L}$ which is closed by Theorem (2.3), part a).

The restrictions $\pi_{P}$ and $\pi_{\mathcal{L}}$ of the natural projections $P \times \mathcal{L} \rightarrow P$ and $P \times \mathcal{L} \rightarrow \mathcal{L}$ are submersions: for a given flag $(p, L)$ choose some point $q \neq p$ on $L$ and let $U$ be an open neighborhood of $p$ in $P$ that does not contain $q$. Putting $\iota: U \rightarrow \mathcal{F}: x \mapsto(x, x \vee q)$, we have $\pi_{P} \circ \iota=\mathrm{id}_{U}$ which shows that $\pi_{P}$ has maximal rank $2 l$ at $(p, L)$. In particular, the fibre $\pi_{\mathcal{L}}^{-1}(L)$ is a submanifold of $\mathcal{F}$ and the restriction $\pi_{L}:=\left.\pi_{P}\right|_{\pi_{\mathcal{L}}^{-1}(L)}$ is a submersion. Because $\pi_{L}$ is a smooth injection between two $l$-dimensional manifolds, the inverse function theorem yields that $\pi_{L}: \pi_{\mathcal{L}}^{-1}(L) \rightarrow L \subset P$ is a homeomorphism and consequently we have the following result.

(2.15) Corollary. For every line $L \in \mathcal{L}$ the restriction $\left.\pi_{P}\right|_{\pi_{\mathcal{L}}^{-1}(L)}$ is a diffeomorphism between the fibre $\pi_{\mathcal{L}}^{-1}(L)$ and the point row $L$.

Analogously, the dual statement holds:

(2.16) Corollary. For every point $q \in P$ the restriction $\left.\pi_{\mathcal{L}}\right|_{\pi_{P}^{-1}(q)}$ is a diffeomorphism of the fibre $\pi_{P}^{-1}(q)$ onto the line pencil $\mathcal{L}_{q}$.

\section{Tangent translation planes}

Let $\mathcal{S}=(P, \mathcal{L}, \mathcal{F})$ be a smooth stable plane of dimension $2 l$. In this chapter we introduce the fundamental concept of a tangent translation plane of the smooth stable plane $\mathcal{S}$. As the name suggests, a tangent translation plane lives on some tangent space $\mathrm{T}_{p} P$. As a main result we prove that the collection $\delta_{p}=\left\{\mathrm{T}_{p} L \mid L \in \mathcal{L}_{p}\right\}$ of vector subspaces of $\mathrm{T}_{p} P$ constitutes a locally compact translation plane $\mathcal{A}_{p}$ on $\mathrm{T}_{p} P$. These translation planes will prove to be one of our most important tools in the investigation of smooth stable planes.

Let us first consider the tangent bundles $\left(\mathrm{T} P, P, \tau_{P}\right),\left(\mathrm{T} \mathcal{L}, \mathcal{L}, \tau_{\mathcal{L}}\right)$, and $\left(\mathrm{TF}, \mathcal{F}, \tau_{\mathcal{F}}\right)$. Since the canonical projection $\pi_{\mathcal{L}}: \mathcal{F} \rightarrow \mathcal{L}$ is a submersion (see the and of the last section), the pullback

$$
\pi_{\mathcal{L}}^{*}(\mathrm{~T} \mathcal{L}):=\left\{(f, v) \in \mathcal{F} \times \mathrm{T} \mathcal{L} \mid \pi_{\mathcal{L}}(f)=\tau_{\mathcal{L}}(v)\right\}
$$


with respect to the diagram

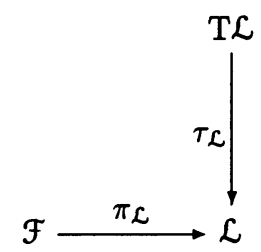

defines a vector bundle $\left(\pi_{\mathcal{L}}^{*}(\mathrm{~T} \mathcal{L}), \mathcal{F}, \tau_{\mathcal{L}}^{\prime}\right)$ with $\tau_{\mathcal{L}}^{\prime}(f, v)=f$, see Husemoller [18], Chap. 3, Prop. 3.1. Moreover, we have the commutative diagram

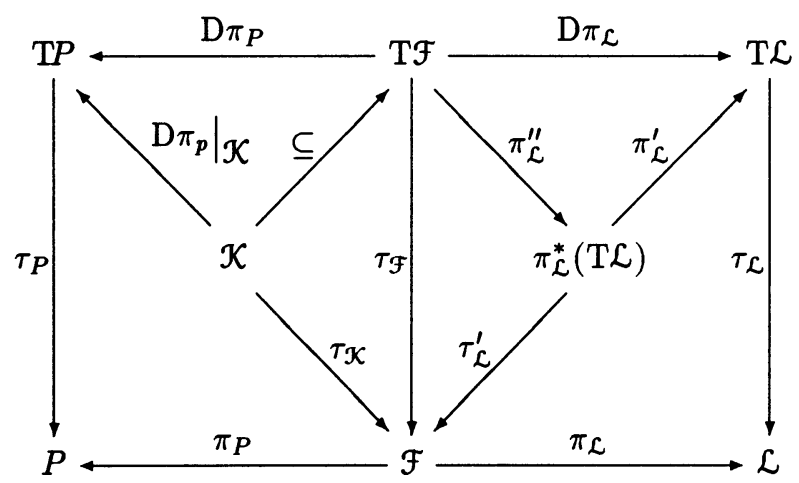

where $\pi_{\mathcal{L}}^{\prime}(f, v)=v$ and $\pi_{\mathcal{L}}^{\prime \prime}(v)=\left(\tau_{\mathcal{F}}(v), \mathrm{D} \pi_{\mathcal{L}}(v)\right)$. The triangles on the left hand side of the diagram will be explained below. For a fixed flag $f=(p, L) \in \mathcal{F}$ we have

$$
\tau_{\mathcal{L}}^{\prime-1}(f)=\left\{(f, v) \in \mathcal{F} \times \mathrm{T} \mathcal{L} \mid \pi_{\mathcal{L}}(f)=\tau_{\mathcal{L}}(v)\right\}=\{f\} \times \tau_{\mathcal{L}}^{-1}\left(\pi_{\mathcal{L}}(f)\right)=\{f\} \times \mathrm{T}_{L} \mathcal{L} .
$$

Since $\pi_{\mathcal{L}}$ is a submersion, the differential $\mathrm{D} \pi_{\mathcal{L}}$ maps every fibre $\mathrm{T}_{f} \mathcal{F}$ of $\tau_{\mathcal{F}}$ onto some fibre $\mathrm{T}_{L} \mathcal{L}$ of $\tau_{\mathcal{L}}$. Hence, by relation $(*)$, we infer that $\pi_{\mathcal{L}}^{\prime \prime}$ maps $\mathrm{T}_{f} \mathcal{F}$ onto $\{f\} \times \mathrm{T}_{L} \mathcal{L}$. Consequently, the triple $\left(\mathcal{X}, \mathcal{F}, \tau_{\mathcal{X}}\right)$, where $\mathcal{X}=\operatorname{ker} \pi_{\mathcal{L}}^{\prime \prime}=\left\{v \in \mathrm{T} \mathcal{F} \mid \pi_{\mathcal{L}}^{\prime \prime}(v) \in \mathcal{F} \times\{0\}\right\}=$ $\left\{v \in \mathrm{TF} \mid \mathrm{D} \pi_{\mathcal{L}}(v)=0\right\}$ and $\tau_{\mathcal{K}}=\left.\tau_{\mathcal{F}}\right|_{\mathcal{K}}$, is a vector subbundle of $\left(\mathrm{TF}, \mathcal{F}, \tau_{\mathcal{F}}\right)$, see Husemoller [18], Chap. 3, Cor. 8.4. The kernel $\mathcal{K}$ consists exactly of those tangent vectors of $\mathrm{TF}$ which are tangent to some fibre of $\pi_{\mathcal{L}}$. Later on we will see that the set $\mathcal{K}$ minus the zero section is mapped by $\mathrm{D} \pi_{P}$ bijectively onto $\mathrm{T} P$ minus the zero section.

Before we proceed, we have to interpose a few facts on (topological) vector bundles. Let $\xi=(E, B, \pi)$ be an $n$-dimensional vector bundle. We write $\mathrm{E}(\xi)^{\times}=E^{\times}$for the total space of $\xi$ with the zero section removed. It is possible to define on $E^{\times}$an equivalence relation $\sim$ by calling two vectors $v_{1}, v_{2} \in E^{\times}$equivalent, if and only if they lie in a common fibre of $\pi$ and span the same one-dimensional subspace there. We write $\mathrm{P} E$ for the quotient space $E^{\times} / \sim$. Let $\pi^{\prime}: E^{\times} \rightarrow \mathrm{P} E$ be the canonical projection. Then the bundle $\mathrm{P} \xi=(\mathrm{P} E, B, \bar{\pi})$, where $\bar{\pi}$ is given by the relation $\pi=\left.\left(\bar{\pi} \circ \pi^{\prime}\right)\right|_{E^{\times}}$, is called the projective bundle associated to $\xi$ (see [18], Chap. 16, (2.1)). Providing PE with the quotient 
topology, the fibres of $\bar{\pi}$ are compact. Thus, the total space $\mathrm{P} E$ is compact if and only if the base space $B$ is compact, cf. Breuning [9], §4, Lemma 2. If $\xi_{1}=\left(E_{1}, B_{1}, \pi_{1}\right)$ and $\xi_{2}=\left(E_{2}, B_{2}, \pi_{2}\right)$ are vector bundles, then every bundle morphism $g: \xi_{1} \rightarrow \xi_{2}$ induces a bundle morphism $\bar{g}$ between the projective bundles $\mathrm{P} \xi_{1}$ and $\mathrm{P} \xi_{2}$, since $g$ is linear on every fibre of $\pi_{1}$. We need the following lemma on vector bundles.

(3.1) Lemma. Let $\xi_{i}=\left(E_{i}, B_{i}, \pi_{i}\right),(i=1,2)$ be two vector bundles, where the base spaces $B_{i}$ are (topological) manifolds and $\operatorname{dim} E_{1}=\operatorname{dim} E_{2}=n$. If $g: \xi_{1} \rightarrow \xi_{2}$ is a bundle morphism such that $g^{\times}:=\left.g\right|_{E_{1}^{\times}}$is injective, then the mapping $g^{\times}: E_{1}^{\times} \rightarrow E_{2}^{\times}$is an open map. If, in addition, $B_{1}$ is compact and $B_{2}$ is connected, then $g^{\times}$is a homeomorphism.

Proof. Passing to the projective bundles $\mathrm{P} \xi_{i}$ associated to $\xi_{i}$, we obtain an injective bundle morphism $\bar{g}: \mathrm{P} \xi_{1} \rightarrow \mathrm{P}_{2}$. By invariance of domain (see Bredon [5], Chap. IV, (19.9)), the maps $\bar{g}$ and $g^{\times}$are open (note that the total spaces $E_{i}$ are topological manifolds as well). According to the remark above, the total space $\mathrm{P} E_{1}$ is a compact $(n-1)$-dimensional topological manifold, and $\mathrm{P} E_{2}$ is a connected manifold of the same dimension. Because the restriction $g^{\times}$is an injection, the induced map $\bar{g}$ is injective on $\mathrm{P} E_{1}$. If $\mathrm{P} E_{1}$ is compact and $\mathrm{P} E_{2}$ is connected, we infer that $\bar{g}$ maps $\mathrm{P} E_{1}$ onto $\mathrm{P} E_{2}$. In order to show that $g^{\times}$is surjective, let $v_{2} \in E_{2}^{\times}$and denote by $\overline{v_{2}}$ the one-dimensional subspace of $E_{2}$ with $v_{2} \in \overline{v_{2}}$. Since $\bar{g}$ is a surjection, there is an element $\overline{v_{1}} \in \mathrm{P} E_{1}$ with $\bar{g}\left(\overline{v_{1}}\right)=\overline{v_{2}}$. By linearity, the map $g$ is a surjection, too.

For the rest of this section, fix some point $p \in P$, and set $\mathcal{B}:=\pi_{P}^{-1}(p)=\{p\} \times \mathcal{L}_{p}$, $\mathcal{G}:=\pi_{\mathcal{L}}^{-1}\left(\mathcal{L}_{p}\right)$, and $\mathcal{K}_{\mathcal{B}}=\left.\mathcal{K}\right|_{\mathcal{B}}$. Via restriction, we obtain from the last diagram

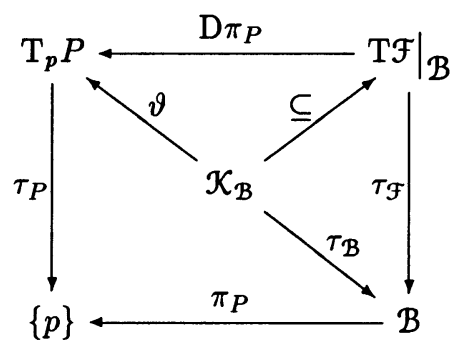

where $\tau_{\mathcal{B}}=\left.\tau_{\mathcal{F}}\right|_{\mathcal{K}_{\mathcal{B}}}$. Using the terminology of Husemoller [18], the next lemma states that $\vartheta$ is an effective Gauss map of the vector bundle $\left(\mathcal{K}_{\mathcal{B}}, \mathcal{B}, \tau_{\mathcal{B}}\right)$ into $\mathrm{T}_{p} P$, cf. [18], Chap. 3, $\S 5$ and [9], §3, p. 22.

(3.2) Lemma. The restriction $\vartheta^{\times}:=\left.\mathrm{D} \pi_{P}\right|_{\left(\mathcal{K}_{\mathcal{B}}\right)^{\times}}:\left(\mathcal{K}_{\mathcal{B}}\right)^{\times} \rightarrow \mathrm{T}_{p} P \backslash\{0\}$ is a smooth homeomorphism with $\vartheta^{\times}\left(\tau_{\mathcal{B}}^{-1}(p, L) \backslash\{0\}\right)=\mathrm{T}_{p} L \backslash\{0\}$ for every line $L \in \mathcal{L}_{p}$.

Proof. Being the restriction of a smooth map to a submanifold of TF, the map $\vartheta^{\times}$is smooth. 
1) In order to show that $\vartheta^{\times}$is injective, let $v_{1}, v_{2} \in\left(\mathcal{K}_{\mathcal{B}}\right)^{\times}$with $\vartheta^{\times}\left(v_{1}\right)=\vartheta^{\times}\left(v_{2}\right)$. Since two distinct point rows through $p$ intersect transversally by Corollary (2.13), and since $v_{1}, v_{2} \neq 0$, there is a flag $(p, L) \in \mathcal{F}$ such that $v_{1}, v_{2} \in \mathrm{T}_{(p, L)} \mathcal{F}$. By Corollary (2.15), the fibre $\pi_{\mathcal{L}}^{-1}(L)$ is mapped by $\pi_{P}$ onto the point row $L \subset P$. Hence, the tangent fibre $\mathrm{D} \pi_{\mathcal{L}}^{-1}\left(\mathrm{~T}_{L} \mathcal{L}\right)$ is mapped injectively into $\mathrm{T} P$ by $\mathrm{D} \pi_{P}$. From $\mathrm{T}_{(p, L)} \mathcal{F} \subseteq \mathrm{D} \pi_{\mathcal{L}}^{-1}\left(\mathrm{~T}_{L} \mathcal{L}\right)$ and $\mathrm{D} \pi_{P}\left(v_{1}\right)=\vartheta^{\times}\left(v_{1}\right)=\vartheta^{\times}\left(v_{2}\right)=\mathrm{D} \pi_{P}\left(v_{2}\right)$, we conclude that $v_{1}=v_{2}$.

2) The vector bundles $\left(\mathrm{T}_{p} P,\{p\},\left.\tau_{P}\right|_{\mathrm{T}_{p} P}\right)$ and $\left(\mathcal{K}_{\mathcal{B}}, \mathcal{B}, \tau_{\mathcal{B}}\right)$ have compact connected manifolds as bases and $\operatorname{dim} \mathrm{T}_{p} P=2 l=l+l=\operatorname{dim} \mathcal{B}+\operatorname{dim} \tau_{\mathcal{B}}^{-1}(b)=\operatorname{dim} \mathcal{K}_{\mathcal{B}}$ holds for every $b \in \mathcal{B}$. By part 1 ), the map $\vartheta$ is a bundle morphism such that $\vartheta^{\times}$is an injection. Thus, the map $\vartheta^{\times}$is a homeomorphism by Lemma (3.1).

3) Since $\vartheta^{\times}$is a bijection by 2) which is linear on the fibres of $\tau_{\mathcal{B}}$ and because of $\operatorname{dim}_{\mathbb{R}} \tau_{\mathcal{B}}^{-1}(p, L)=\operatorname{dim}_{\mathbb{R}} \mathrm{T}_{p} L$, it suffices to show that $\vartheta^{\times}\left(\tau_{\mathcal{B}}^{-1}(p, L) \backslash\{0\}\right) \subseteq \mathrm{T}_{p} L \backslash\{0\}$ holds for every line $L \in \mathcal{L}_{p}$. Let $(p, L) \in \mathcal{B}$ and select a vector $v \in \tau_{\mathcal{B}}^{-1}(p, L) \backslash\{0\}$. By definition of $\mathcal{K}_{\mathcal{B}}$, the vector $v$ is tangent to the fibre $\pi_{\mathcal{L}}^{-1}(L)$. Since this fibre is mapped into $P$ by the diffeomorphism $\left.\pi_{P}\right|_{\pi_{\mathcal{L}}^{-1}(L)}$, the image of $v$ under the map D $\pi_{P}$ lies in the tangent space $\mathrm{T}_{p} L \backslash\{0\}$. This completes the proof of the Lemma.

We call the Gauss map $\vartheta_{p}=\vartheta^{\times}$the characteristic map of $\mathcal{S}$ at the point $p$, and we refer to the restriction $\left.\mathrm{D} \pi_{P}\right|_{\mathcal{K}}$ as the (global) characteristic map of $\mathcal{S}$.

(3.3) Definition. Let $V$ be a real vector space of dimension $2 m$. A family $X$ of $m$ dimensional subspaces of $V$ is called a spread in $V$, if and only if the properties

(i) $\bigcup x=V$,

(ii) $X_{1} \oplus X_{2}=V$ for every pair of distinct elements $X_{1}, X_{2}$ of $x$ are satisfied.

Every point row $L \in \mathcal{L}_{p}$ is a closed submanifold of $P$ by Theorem (2.6) and their tangent spaces $\mathrm{T}_{p} L$ at $p$ are $l$-dimensional subspaces of $\mathrm{T}_{p} P$. Since two distinct lines $K, L$ of $\mathcal{L}_{p}$ are transversal by Corollary (2.13), the tangent space $\mathrm{T}_{p} P$ decomposes as $\mathrm{T}_{p} P=\mathrm{T}_{p} K \oplus \mathrm{T}_{p} L$. We call $\mathcal{S}_{p}:=\left\{\mathrm{T}_{p} L \mid L \in \mathcal{L}_{p}\right\}$ the tangent spread of $\mathcal{S}$ at $p$. By Lemma (3.2) and the remark preceeding it, we get $\bigcup \mathcal{S}_{p}=\mathrm{T}_{p} P$, and thus we have indeed:

(3.4) Proposition. The set $\mathcal{S}_{p}$ is a spread in $\mathrm{T}_{p} P$.

Every spread of a vector space $V$ defines an (affine) translation plane, see André [1] or Bruck/Bose [11]. Thus, Proposition (3.4) shows that the tangent space $\mathrm{T}_{p} P$ is an affine translation plane $\mathcal{A}_{p}$ which is defined by the spread $\mathcal{S}_{p}$. In fact, we will even prove the following theorem. 
(3.5) Theorem. The tangent translation planes $\mathcal{A}_{p}$ of a smooth stable plane $\mathcal{S}$ are locally compact connected topological affine translation planes.

Since every locally compact connected affine translation plane is coordinatized by a locally compact connected ternary field (see [36], 7.15, 71.6, or [14], 4.2), the theorem above yields the following result (compare also Löwen [31]).

(3.6) Corollary. The projective closures $\mathcal{P}_{p}$ of the tangent planes $\mathcal{A}_{p}$ are compact connected projective translation planes.

We will base the proof of Theorem (3.5) on the first Linearization Theorem (3.9). Recall that $\mathcal{O}_{\wedge}$ is the domain of the intersection map $\wedge$.

(3.7) Lemma. The inverse image $\mathcal{S}=\pi_{\mathcal{L}}^{-1}\left(\mathcal{L}_{p}\right)$ is a submanifold of $\mathcal{F}$.

Proof. By Theorem (2.6), the line pencil $\mathcal{L}_{p}$ is a submanifold of $\mathcal{L}$, and thus $P \times \mathcal{L}_{p}$ is a submanifold of $P \times \mathcal{L}$. Since $\mathcal{G} \subseteq P \times \mathcal{L}_{p}$, it suffices to show that $\mathcal{G}$ is a submanifold of $P \times \mathcal{L}_{p}$. In order to verify this, we will again utilize Lemma (2.5). Let $(q, L) \in P \times \mathcal{L}_{p}$. Choose some point $o \in P \backslash L$ and let $U$ and $\mathcal{V}$ be connected open neighborhoods of $q$ and $L$, respectively, such that $\{x \vee o \mid x \in U\} \times \mathcal{V} \subseteq \mathcal{O}_{\wedge}$ holds. Considering as in Theorem (2.14) the smooth retraction

$$
f: U \times \mathcal{V} \rightarrow P \times \mathcal{L}_{p}:(r, K) \mapsto((r \vee o) \wedge K, K)
$$

onto $\mathcal{G} \cap(U \times \mathcal{V})$, we conclude that $\mathcal{G} \cap(U \times \mathcal{V})$ is a submanifold of $U \times \mathcal{V}$. Since $\mathcal{G} \backslash \mathcal{B}$ is diffeomorphic to $P \backslash\{p\}$ via the projection $(r, K) \mapsto r$, we infer by Theorem (2.3), part d) that all these local submanifolds $\mathcal{G} \cap(U \times \mathcal{V})$ are of uniform dimension. Hence, by Lemma (2.5) we infer that $\mathcal{G}$ is a submanifold of $P \times \mathcal{L}_{p}$.

In the next corollary we use the notion of a (smooth) microbundle, cp. Milnor, [32] and the book of Kirby and Siebenmann [20], Essay IV, $§ 1$.

(3.8) Definition. A quadruple $(M, N, \kappa, \iota)$ is called a smooth $n$-microbundle, if $M$ and $N$ are smooth manifolds and $\kappa: M \rightarrow N$ and $\iota: N \rightarrow M$ are smooth maps such that the following axioms are satisfied:

(i) $\kappa \circ \iota=\mathbb{1}_{N}$,

(ii) For any $x \in N$ there are open neighborhoods $U$ of $x$ and $V$ of $\iota(x)$ such that $\iota(U) \subseteq V, \kappa(V) \subseteq U$ and the diagram above commutes for some homeomorphism $h: V \rightarrow U \times \mathbb{R}^{n}$

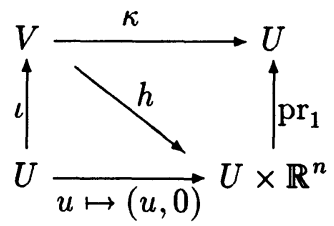


(3.9) Corollary. The quadruple $(\mathcal{G}, \mathcal{B}, \kappa, \iota)$, where $\iota: \mathcal{B} \hookrightarrow \mathcal{G}$ is the inclusion map and $\kappa: \mathcal{G} \rightarrow \mathcal{B}:(q, L) \mapsto(p, L)$, is a smooth l-dimensional microbundle.

Proof. Clearly, we have $\kappa \circ \iota=\mathbb{1}_{\mathcal{B}}$. Let $(p, L) \in \mathcal{B}$ and choose some line $L^{\prime} \in \mathcal{L}_{p} \backslash\{L\}$. Select neighborhoods $U \subseteq P$ of $p$ and $\mathcal{U} \subseteq \mathcal{L}$ of $L$ according to Lemma (2.11). The set $\mathcal{V}:=\{p\} \times\left(\mathcal{U} \cap \mathcal{L}_{p}\right)$ is an open neighborhood of $(p, L)$ in $\mathcal{B}$ and $\mathcal{W}:=(U \times \mathcal{U}) \cap \mathcal{G}$ is an open neighborhood of $(p, L)$ in $\mathcal{G}$. The map

$$
h: \mathcal{V} \times(U \cap L) \rightarrow \mathcal{W} \cap \kappa^{-1}(\mathcal{V}):((p, K), q) \mapsto\left(\left(q \vee q^{\prime}\right) \wedge K, K\right)
$$

where $q^{\prime} \in L^{\prime}$ corresponds to $q_{2}$ in Lemma (2.11), is a diffeomorphism by Lemma (2.11)(iii). It satisfies the relations

$$
\kappa \circ h((p, K), q)=\kappa\left(\left(q \vee q^{\prime}\right) \wedge K, K\right)=(p, K)
$$

and

$$
h((p, K), p)=(p, K) .
$$

Since $U \cap L$ is diffeomorphic to $\mathbb{R}^{l}$ by Lemma (2.11)(ii), this shows that $h$ is a smooth local trivialization of $(\mathcal{G}, \mathcal{B}, \kappa)$ and hence $(\mathcal{G}, \mathcal{B}, \kappa, \iota)$ is a smooth microbundle.

By the Kister-Mazur theorem (see Kister [21], Kuiper/Lashof [23], and Siebenmann/ Guillou/Hähl [37]), there is an open neighborhood $\mathcal{W}$ of $\mathcal{B}$ in $\mathcal{G}$ such that $\xi=(\mathcal{W}, \mathcal{B}, \kappa \mid \mathcal{W})$ is a locally trivial $\mathbb{R}^{l}$-bundle with zero section $\iota$ and structure group Diff $0\left(\mathbb{R}^{l}\right)$ consisting of all origin-preserving diffeomorphisms of $\mathbb{R}^{l}$. Since $O_{l} \mathbb{R}$ is a deformation retract of $\operatorname{Diff}\left(\mathbb{R}^{l}\right)$ (see Stewart [38]), we may reduce the structure group of $\xi$ to $O_{l} \mathbb{R}$. In this way, the bundle $\xi$ becomes a smooth $l$-dimensional vector bundle. Moreover, we have $\operatorname{dim} \mathfrak{G}=$ $\operatorname{dim} \mathcal{B}+\operatorname{dim} \mathbb{R}^{l}=2 l$, and thus the set $\mathcal{W}$ is a tubular neighborhood of $\mathcal{B}$ (Kosinski [22], III, (2.4)). Since any two tubular neighborhoods of $\mathcal{B}$ in $\mathcal{G}$ are isomorphic, the bundle $\xi$ is isomorphic to the normal bundle $\nu \mathcal{B}$ of $\mathcal{B}$ in $\mathcal{G}$, see [22], (3.2) and (2.3). We will denote this isomorphism by $\beta_{1}$. Note that $\xi$ and $\nu \mathcal{B}$ are isomorphic smooth vector bundles, hence $\beta_{1}$ induces a diffeomorphism between their total spaces, cp. [22], p. 48, Ex. Providing $\mathcal{G}$ with a Riemannian metric, we may represent $\nu \mathcal{B}$ as the orthogonal bundle to $\mathrm{TB}$ in $\left.\mathrm{TG}\right|_{\mathcal{B}}$. The restriction $\left.\pi_{\mathcal{L}}\right|_{\mathcal{B}}: \mathcal{B} \rightarrow \mathcal{L}$ is an embedding and thus $\left.\mathrm{D} \pi_{\mathcal{L}}\right|_{\mathrm{T} \mathcal{B}}: \mathrm{TB} \rightarrow \mathrm{T} \mathcal{L}$ is an injection. Recalling that $\mathcal{K}=\left\{v \in \mathrm{TF} \mid \mathrm{D} \pi_{\mathcal{L}}(v)=0\right\}$, we thus have $\mathrm{T}_{(p, L)} \mathcal{B} \cap \mathcal{K}_{(p, L)}=\{0\}$ for every line $L \in \mathcal{L}_{p}$. In particular, no vector of $\mathcal{K}_{(p, L)}$ is orthogonal to $\nu_{(p, L)} \mathcal{B}$. Since both $\nu \mathcal{B}$ and $\mathcal{K}_{\mathcal{B}}$ are $l$-dimensional vector bundles over $\mathcal{B}$, orthogonal projection in each fibre gives rise to a bundle isomorphism $\beta_{2}$ between $\nu \mathcal{B}$ and $\mathcal{K}_{\mathcal{B}}$. According to Lemma (3.2), the composition

$$
\beta: \mathcal{W} \backslash \mathcal{B} \stackrel{\beta_{1}}{\rightarrow} \mathrm{E}(\nu \mathcal{B})^{\times} \stackrel{\beta_{2}}{\rightarrow} \mathrm{E}\left(\mathcal{K}_{\mathcal{B}}\right)^{\times} \stackrel{\vartheta^{\times}}{\rightarrow} \mathrm{T}_{p} P \backslash\{0\}
$$


is a smooth homeomorphism. The following two theorems are the main result of this section. They state that a smooth stable plane can be linearized in some neighborhood of each point.

(3.10) First Linearization Theorem. There exists an open neighborhood $U$ of $p$ in $P$ and a smooth homeomorphism $\lambda: U \backslash\{p\} \rightarrow \mathrm{T}_{p} P \backslash\{0\}$ such that $\lambda(L \cap U \backslash\{p\})=\mathrm{T}_{p} L \backslash\{0\}$ holds for every line $L \in \mathcal{L}_{p}$.

Proof. Since the projection $\mathrm{pr}_{1}: \mathcal{G} \backslash \mathcal{B} \rightarrow P \backslash\{p\}:(q, L) \mapsto q$ is a smooth homeomorphism and because $\mathcal{W}$ is an open neighborhood of $\mathcal{B}$ in $\mathcal{G}$, the set $U:=\operatorname{pr}_{1}(\mathcal{W})$ is an open neighborhood of $p$ in $P$. Moreover, the map $\alpha: U \backslash\{p\} \rightarrow \mathcal{W} \backslash \mathcal{B}: q \mapsto(q, p \vee q)$ is a smooth bijection having $\mathrm{pr}_{1}$ as a smooth inverse map. Hence, the composition $\lambda:=\beta \circ \alpha$ : $U \backslash\{p\} \rightarrow \mathrm{T}_{p} P \backslash\{0\}$ is a smooth homeomorphism, too. For every line $L \in \mathcal{L}_{p}$ we have $\alpha(L \cap U \backslash\{p\})=((L \cap U) \backslash\{p\}) \times\{L\}$ and the composition $\beta_{2} \circ \beta_{1}$ maps the latter set one-to-one onto $\tau_{\mathcal{B}}^{-1}(p, L) \backslash\{0\}$. Hence, we have $\lambda(L \cap U \backslash\{p\})=\mathrm{T}_{p} L \backslash\{0\}$ according to Lemma (3.2).

Remark. The smooth homeomorphism $\lambda$ we constructed can be regarded as the inverse map of some special exponential map which maps the tangent spaces of point rows $L$ through $p$ into $L$. This is quite similar to the situation for symmetric planes, see Löwen, $[29]$.

(3.11) Lemma. Let $h$ be a homeomorphism of $R:=\mathbb{R}^{n} \backslash\{0\}$ onto itself, where $n \geq 2$, and let $S:=R \cup\{0\} \cup\{\infty\} \approx \mathbb{S}_{n}$ be the two-point compactification of $R$. Then the map $h$ can be extended to a homeomorphism $H: S \rightarrow S$.

Proof. Let $\Sigma_{1} \subseteq R$ be an $(n-1)$-sphere. Then $\Sigma_{2}:=h\left(\Sigma_{1}\right)$ is an $(n-1)$-sphere too. By the generalized Jordan curve theorem (see Bredon [5], Chap. IV, §19, e.g.) the complements $S \backslash \Sigma_{i}$ consist of exactly two connected components $K_{i}, L_{i}$, where we may assume that $0 \in K_{i}$. Since $n \geq 2$, the sets $K_{i}^{*}:=K_{i} \backslash\{0\}$ and $L_{i}^{*}:=L_{i} \backslash\{\infty\}$ are the connected components of $R$. Thus we have $h\left(K_{1}^{*}\right), h\left(L_{1}^{*}\right) \in\left\{K_{2}^{*}, L_{2}^{*}\right\}$. Suppose that $h\left(K_{1}^{*}\right)=L_{2}^{*}$. Extending the map $h$ by $H(0)=\infty$ and $H(\infty)=0$ to $S$, it remains to show that $H: S \rightarrow S$ is continuous at the points 0 and $\infty$. Choose a sequence $\left(x_{n}\right)_{n \in \mathbb{N}}$ in $S$ that converges to 0 . Since $n$-spheres are locally connected, we may assume that this sequence lies in $K_{1}$. The sequence $\left(h\left(x_{n}\right)\right)_{n \in \mathbb{N}}$ is thus contained in the compact set $L_{2} \cup \Sigma_{2}$. In particular, the sequence $\left(h\left(x_{n}\right)\right)_{n \in \mathbb{N}}$ has an accumulation point in $L_{2} \cup \Sigma_{2}$. Since $h: R \rightarrow R$ is a homeomorphism, every accumulation point of the sequence above must be $\infty$. Hence, the map $H$ is continuous at the point 0 . Analoguously, one proves that $H$ is continuous at $\infty$ and that $H^{-1}$ is continuous. For the case $h\left(K_{1}^{*}\right)=K_{2}^{*}$, the proof runs as before.

From Theorem (3.10) and the previous lemma we immediately get the second Linearization Theorem. 
(3.12) Second Linearization Theorem. There exists an open neighborhood $U$ of $p$ in $P$ and a homeomorphism $\lambda: U \rightarrow \mathrm{T}_{p} P$ such that $\lambda(L \cap U)=\mathrm{T}_{p} L$ holds for every line $L \in \mathcal{L}_{p}$.

Proof. By Lemma (3.11) we can extend the homeomorphism $\lambda: U \backslash\{p\} \rightarrow \mathrm{T}_{p} P \backslash\{0\}$ to some homeomorphism $\tilde{\lambda}: U \cup\{\infty\} \rightarrow \mathrm{T}_{p} P \cup\{\infty\}$. If $\widetilde{\lambda}(p)=\infty$, we take a rotation $\varphi$ of the $l$-sphere $\mathrm{T}_{p} P \cup\{\infty\}$ which interchanges the points 0 and $\infty$. Otherwise we take the identity for $\varphi$. Then the composition $\varphi \circ \widetilde{\lambda}$ obviously satisfies the assertion of the theorem.

In order to prove Theorem (3.5), we have to check that the tangential spread $\mathcal{S}_{p}$ is compact with respect to the Grassmann topology, see Löwen [31] and [36], 64.4d.

(3.13) Corollary. The tangent spread $\mathcal{S}_{p}$ is a compact subset of the Grassmann manifold $\mathrm{G}_{l}\left(\mathbb{R}^{2 l}\right)$.

Proof. Let $\left(\mathrm{T}_{p} L_{\nu}\right)_{\nu \in \mathbb{N}}$ be a sequence of spread elements of $\mathcal{S}_{p}$ converging to an $l$-dimensional vector subspace $V$ of $\mathbb{R}^{2 l}$. We have to show that $V \in \mathcal{S}_{p}$. Let $q \in V \backslash\{0\}$. There are vectors $q_{\nu} \in \mathrm{T}_{p} L_{\nu} \backslash\{0\}$ such that the sequence $\left(q_{\nu}\right)_{\nu \in \mathbb{N}}$ converges to $q$ in $\mathbb{R}^{2 l}$. By Theorem (3.10), the sequence $\lambda^{-1}\left(q_{\nu}\right) \subseteq U \backslash\{p\}$ converges to $\lambda^{-1}(q)$, and hence $\lambda^{-1}\left(q_{\nu}\right) \vee p=L_{\nu}$ converges to $L:=\lambda^{-1}(q) \vee p$ in $\mathcal{L}$. Thus we have

$$
q=\lambda\left(\lambda^{-1}(q)\right) \in \lambda(L \cap U \backslash\{p\})=\mathrm{T}_{p} L \backslash\{0\}
$$

and we conclude that $V$ is contained in $\mathrm{T}_{p} L \backslash\{0\}$. Since both subspaces $V$ and $\mathrm{T}_{p} L$ have the same dimension $l$, the claim follows.

(3.14) Corollary. For every point $p \in P$ the map $\sigma: \mathcal{L}_{p} \rightarrow \mathcal{S}_{p}: L \mapsto \mathrm{T}_{p} L$ is a homeomorphism.

Proof. Clearly, the map $\sigma$ is a bijection. For $q \in \mathrm{T}_{p} P \backslash\{0\}$ we denote by $S_{q}$ the unique spread element of $S_{p}$ that contains $q$. Since the affine tangent plane $\mathcal{A}_{p}$ is a topological plane by (3.5), the join map $\tau: \mathrm{T}_{p} P \backslash\{0\} \rightarrow \mathcal{S}_{p}: q \mapsto S_{q}$ is a continuous surjection. Hence, the composition $\sigma \circ \vee_{p}=\tau \circ \lambda$ is continuous on $U \backslash\{p\}$. Since $\vee_{p}$ is an identification map, we conclude that $\sigma$ is continuous, too. Because the line pencil $\mathcal{L}_{p}$ is compact, the map $\sigma$ is in fact a homeomorphism.

\section{Tangent shear planes}

In the case of a smooth projective plane the results of the preceeding section can canonically be dualized in order to get a tangent translation plane on the tangent space $\mathrm{T}_{L} \mathcal{L}$ of any line $L \in \mathcal{L}$. For a smooth stable plane $\mathcal{S}$, however, the set $\mathcal{S}_{L}:=\left\{\mathrm{T}_{L} \mathcal{L}_{p} \mid p \in L\right\}$ 
where $L$ is some line of $\mathcal{S}$, may fail to satisfy condition (i) of Definition (3.3). Nevertheless, the set $\mathcal{S}_{L}$ still satisfies property (ii) of Definition (3.3). Such a set is called a partial spread, cf. Löwe [27], (2.2). H. Löwe has shown that each partial spread which is a $l$-dimensional (topological) submanifold of the Grassmannian $G_{l}\left(\mathbb{R}^{2 l}\right)$ gives rise to a stable plane. Such stable planes which turn out to be shear planes can be regarded as a generalization of translation planes. The following definition shows why.

(4.1) Definition. Let $\mathcal{S}=(P, \mathcal{L}, \mathcal{F})$ be a stable plane. A subgroup $\Gamma$ of $\operatorname{Aut}(\mathcal{S})$ is called quasi-perspective or straight, if every orbit of $\Gamma$ is contained in some point row of $\mathcal{S}$. The stable plane $\mathcal{S}$ is called a shear plane if there exists a quasi-perspective closed subgroup $\Gamma \cong \mathbb{R}^{2 l}$ that acts on $P$ without fixed points.

Note that for an affine translation plane the subgroup $\Gamma$ is the translation group. Other examples of affine shear planes are the so-called shift planes, where $\Gamma$ possesses a subgroup $T \cong \mathbb{R}^{l}$ of translations with common axis (= line at infinity) and common center, see $[36], \S 74$.

In this section we shall show that for each line $L$ the set $\mathcal{S}_{L}:=\left\{\mathrm{T}_{L} \mathcal{L}_{p} \mid p \in L\right\}$ defines a shear plane. We start by briefly describing how shear planes can be constructed out of partial spreads, cf. Löwe, [27], Section 3. Let $H$ be some hyperplane of $\mathbb{R}^{2 l+1}$ and let $X \subseteq \mathrm{G}_{l}(H)$ be a partial spread which is an l-dimensional (topological) submanifold of $\mathrm{G}_{l}(H)$ with respect to the induced topology. We shall call such a partial spread proper. Then a stable plane $\mathcal{S}=(P, \mathcal{L}, \mathcal{F})$ is defined as follows. Take $P=$ $\left\{p \in \mathrm{G}_{l+1}\left(\mathbb{R}^{2 l+1}\right) \mid p \cap H \in X\right\}$ with the induced topology, set $\mathcal{L}=X \cup \mathrm{G}_{1}\left(\mathbb{R}^{2 l+1}\right) \backslash \mathrm{G}_{1}(H)$ and define $\mathcal{F}=\{(p, L) \in P \times \mathcal{L} \mid L \leq p\}$. By a theorem of Löwe ([27], Theorem 1) the incidence structure $\mathcal{S}$ is a shear plane with group $\Gamma=\left\{\gamma_{a} \mid a \in H\right\}$, where $\gamma_{a}$ is the linear map which fixes $H$ pointwise and maps some fixed vector $e \notin H$ to $e+a$. So what we have to prove is that $\delta_{L}$ is a proper partial spread. In order to do this, we shall generalize the results of the last section. For a start we consider the double square diagram at the beginning of the Section 3 with the rôles of $\mathcal{L}$ and $P$ interchanged. We denote the resulting diagram by (D). Fixing some line $L \in \mathcal{L}$ and setting $\mathcal{B}:=\pi_{\mathcal{L}}^{-1}(L)=\{L\} \times L, \mathcal{E}:=\pi_{P}^{-1}(L)$, and $\mathcal{K}_{\mathcal{B}}=\left.\mathcal{K}\right|_{\mathcal{B}}$, we get from diagram (D)

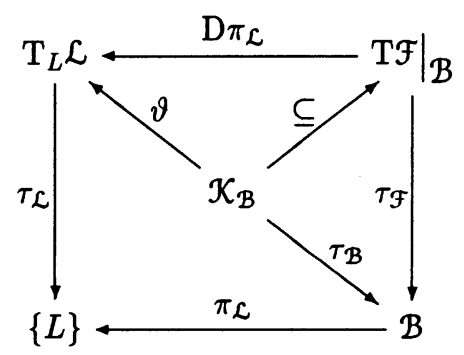

where $\tau_{\mathcal{B}}=\left.\tau_{\mathcal{F}}\right|_{\mathcal{X}_{\mathcal{B}}}$. As in Section 3, it turns out that the map $\vartheta$ is an injection; for the proof use corollaries (2.13) and (2.16). Moreover, the vector bundles $\left(\mathrm{T}_{L} \mathcal{L},\{L\},\left.\tau_{\mathcal{L}}\right|_{\mathrm{T}_{L} \mathcal{L}}\right)$ 
and $\left(\mathcal{K}_{\mathcal{B}}, \mathcal{B}, \tau_{\mathcal{B}}\right)$ both have $2 l$-dimensional manifolds as total spaces. Applying Lemma (3.1) to $\vartheta$ we get that $\vartheta^{\times}:=\left.\mathrm{D} \pi_{\mathcal{L}}\right|_{\left(\mathcal{K}_{\mathcal{B}}\right)^{\times}}:\left(\mathcal{K}_{\mathcal{B}}\right)^{\times} \rightarrow \mathrm{T}_{L} \mathcal{L} \backslash\{0\}$ is a smooth topological embedding. For $(p, L) \in \mathcal{B}$ any non-zero vector $v \in \tau_{\mathcal{B}}^{-1}(p, L)$ is tangent to the fibre $\tau_{P}^{-1}\left(\mathcal{L}_{p}\right)$ by definition of $\mathcal{K}_{\mathcal{B}}$. Since this fibre is mapped into $\mathcal{L}$ by the projection $\pi_{\mathcal{L}}$ (Corollary (2.16)), we have $\vartheta^{\times}\left(\tau_{\mathcal{B}}^{-1}(p, L) \backslash\{0\}\right) \subseteq \mathrm{T}_{L} \mathcal{L}_{p} \backslash\{0\}$ for every point $p \in L$. In fact, equality holds, for $\vartheta$ is a linear bijection and $\operatorname{dim}_{\mathbb{R}} \tau_{\mathcal{B}}^{-1}(p, L)=\operatorname{dim}_{\mathbb{R}} \mathrm{T}_{L} \mathcal{L}_{p}$. Thus we have proved the following lemma.

(4.2) Lemma. The restriction $\vartheta^{\times}:=\left.\mathrm{D} \pi_{\mathcal{L}}\right|_{\left(\mathcal{K}_{\mathcal{B}}\right)^{\times}}:\left(\mathcal{K}_{\mathcal{B}}\right)^{\times} \rightarrow \mathrm{T}_{L} \mathcal{L} \backslash\{0\}$ is a smooth topological embedding with $\vartheta^{\times}\left(\tau_{\mathcal{B}}^{-1}(p, L) \backslash\{0\}\right)=\mathrm{T}_{L} \mathcal{L}_{p} \backslash\{0\}$ for every point $p \in L$.

Using Lemma (2.12) instead of Lemma (2.11), we get as in Section 3:

(4.3) Proposition. The inverse image $\mathcal{E}:=\pi_{P}^{-1}(L)$ is a submanifold of $\mathcal{F}$. The quadruple $(\mathcal{E}, \mathcal{B}, \rho, \iota)$, where $\iota: \mathcal{B} \hookrightarrow \mathcal{E}$ is the inclusion map and $\rho: \mathcal{E} \rightarrow \mathcal{B}:(p, K) \mapsto(p, L)$, is a smooth l-dimensional microbundle.

There is an open neighborhood $\mathcal{W}$ of $\mathcal{B}$ in $\mathcal{E}$ such that $\xi=\left(\mathcal{W}, \mathcal{B},\left.\rho\right|_{\mathcal{W}}\right)$ is a locally trivial bundle and we obtain a smooth topological embedding

$$
\beta^{\prime}: \mathcal{W} \backslash \mathcal{B} \stackrel{\beta_{1}^{\prime}}{\longrightarrow} \mathrm{E}(\nu \mathcal{B})^{\times} \stackrel{\beta_{2}^{\prime}}{\longrightarrow} \mathrm{E}\left(\mathcal{K}_{\mathcal{B}}\right)^{\times} \stackrel{\vartheta^{\times}}{\longrightarrow} \mathrm{T}_{L} \mathcal{L} \backslash\{0\}
$$

where $\beta_{1}^{\prime}$ and $\beta_{2}^{\prime}$ are diffeomorphisms. Using this result we can prove a similar linearization theorem as in the last section.

(4.4) Third Linearization Theorem. There exists an open neighborhood $\mathcal{U}$ of $L$ in $\mathcal{L}$ and a smooth topological embedding $\lambda^{\prime}: \mathcal{U} \backslash\{L\} \rightarrow \mathrm{T}_{L} \mathcal{L} \backslash\{0\}$ such that $\lambda\left(\mathcal{L}_{p} \cap \mathcal{U} \backslash\{L\}\right)=$ $\mathrm{T}_{L} \mathcal{L}_{p} \backslash\{0\}$ holds for every point $p \in L$.

Now we are going to prove that the partial spread $\mathcal{S}_{L}$ defines a shear plane.

(4.5) Corollary. For every line $L \in \mathcal{L}$ the map $\sigma^{\prime}: L \rightarrow \mathcal{S}_{L}: p \mapsto \mathrm{T}_{L} \mathcal{L}_{p}$ is a topological embedding, where $\mathcal{S}_{L}$ is taken with the induced topology of the Grassmannian $\mathrm{G}_{l}\left(\mathrm{~T}_{L} \mathcal{L}\right)$, and the incidence structure $\mathcal{P}\left(\mathcal{S}_{L}\right)$ is a shear plane.

Proof. Since line pencils meet transversally in $\mathcal{L}$ by Corollary (2.13), the map $\sigma^{\prime}$ is injective. Let $\left(\mathcal{L}_{p_{n}}\right)$ be a sequence of line pencils with $\left(p_{n}\right) \subseteq L$ converging to some point $p \in L$. Since $G_{l}\left(T_{L} \mathcal{L}\right)$ is compact, we may assume that $T_{L} \mathcal{L}_{p_{n}}$ converges to some vector space $V \in \mathrm{G}_{l}\left(\mathrm{~T}_{L} \mathcal{L}\right)$. Let $\lambda^{\prime}: \mathcal{U} \backslash\{L\} \rightarrow \mathrm{T}_{L} \mathcal{L} \backslash\{0\}$ be the map of Theorem (4.4). Fix some line $K \in \mathcal{U}$ through $p$ and select a sequence $\left(K_{n}\right) \subseteq \mathcal{U}$ of lines through $p_{n}$ which converges to $K$ in $\mathcal{L}$. Then $\lambda^{\prime}\left(K_{n}\right) \in \mathrm{T}_{L} \mathcal{L}_{p_{n}}$ converges to $\lambda^{\prime}(K) \in \mathrm{T}_{L} \mathcal{L}_{p}$. Thus we have $\lambda^{\prime}(K) \in V$ 
for every $K \in \mathcal{L}_{p}$. From $\mathrm{T}_{L} \mathcal{L}_{p} \subseteq V$ and $\operatorname{dim} \mathrm{T}_{L} \mathcal{L}_{p}=\operatorname{dim} V$ we conclude that $\mathrm{T}_{L} \mathcal{L}_{p}$ and $V$ coincide. Hence, the map $\sigma^{\prime}$ is continuous. Conversely, let $\left(\mathrm{T}_{L} \mathcal{L}_{p_{n}}\right)$ be a sequence in $\mathrm{G}_{l}\left(\mathrm{~T}_{L} \mathcal{L}\right)$ that converges to $\mathrm{T}_{L} \mathcal{L}_{p}$. Select some non-zero vector $w \in \mathrm{T}_{L} \mathcal{L}_{p}$ as well as a sequence $\left(w_{n}\right)$ of elements $w_{n} \in \mathrm{T}_{L} \mathcal{L}_{p_{n}} \backslash\{0\}$ that converges to $w$. Then $\lambda^{\prime-1}\left(w_{n}\right)$ converges to $\lambda^{\prime-1}(w) \in \mathcal{U} \subseteq \mathcal{L}$. Since $\lambda^{\prime-1}\left(w_{n}\right) \in \mathcal{L}_{p_{n}}$ and $\lambda^{\prime-1}(w) \in \mathcal{L}_{p}$ by Theorem (4.4), the continuity of the intersection map implies that $p_{n}=\lambda^{\prime-1}\left(w_{n}\right) \wedge L$ converges to $\lambda^{\prime-1}(w) \wedge L=p$ in $L \subseteq P$. Now the second claim follows from Löwe, [27], Theorem 1 .

\section{Homogeneity of smooth projective planes}

In our last section we shall prove some homogeneity properties of a smooth projective plane $\mathcal{P}=(P, \mathcal{L}, \mathcal{F})$. Let $n=\operatorname{dim} P=2 l$. We start by showing that any two point rows are embedded equivalently into the point space $P$. More precisely, we have the following lemma.

(5.1) Lemma. Let $L_{0}$ and $L_{1}$ be two point rows of $\mathcal{P}$. Then there is a diffeotopy $F: P \times[0,1] \rightarrow P$ with $F_{0}=\mathrm{id}_{P}$ and $F_{1}\left(L_{0}\right)=L_{1}$.

Proof. Let $p=L_{0} \wedge L_{1}$. The line pencil $\mathcal{L}_{p}$ is a smooth manifold homeomorphic to the $l$-sphere $([4],(4.4))$. Hence there is a smooth path $\gamma:[0,1] \rightarrow \mathcal{L}_{p}$ from $L_{0}$ to $L_{1}$ with $\gamma([0,1]) \neq \mathcal{L}_{p}$. Choose a point $q \in P$ which is not incident with a line of $\gamma([0,1])$. Consider the isotopy

$$
f: L_{0} \times[0,1] \rightarrow P:(x, t) \mapsto(x \vee q) \wedge \gamma(t) .
$$

Clearly, $f_{0}$ is the identity on $L_{0}, f_{1}\left(L_{0}\right)=L_{1}$, and every map $f_{t}$ is an embedding, since every point row of $\mathcal{P}$ is a compact submanifold of $P$ by Theorem (2.6). By the isotopy extension theorem (see e.g. Hirsch, [17], §8), the isotopy $f$ can be extended to a diffeotopy $F$ on $P$ with $F_{0}=\mathrm{id}_{P}$. This proves the lemma.

Of course, the dual statement of Lemma (5.1) is also true, i.e. in $\mathcal{L}$ any two line pencils are embedded equivalently. From now on we shall always omit the formulations (and the proofs) of the dual statements.

(5.2) Lemma. For any point row $L$ and every point $p$ not on $L$ the bundle $\xi_{p, L}=$ $\left(P \backslash\{p\}, L, \wedge_{L} \circ \vee_{p}\right)$ is a smooth locally trivial $\mathbb{R}^{\prime}$-bundle with zero section $\iota: L \hookrightarrow P \backslash\{p\}$.

Proof. The map $\pi=\Lambda_{L} \circ \vee_{p}$ is smooth. The fibres of $\pi$ are the point rows through $p$ minus the point $p$ itself. Thus, the fibres of $\pi$ are diffeomorphic to $\mathbb{R}^{l}$, see [4], (4.4). Using Lemma (2.11) it is easy to see that $\xi_{p, L}$ is locally trivial, cp. the proof of Corollary (3.9). Since $\pi$ is the identity on $L$, the inclusion map $\iota: L \hookrightarrow P \backslash\{p\}$ is a smooth zero section of $\xi_{p, L}$. 
Reducing the structure group of $\xi_{p, L}$ from $\operatorname{Diff}_{0}\left(\mathbb{R}^{l}\right)$ to $\mathrm{GL}_{l} \mathbb{R}$ (see Stewart [38]), the $\mathbb{R}^{l}$-bundle $\xi_{p, L}$ becomes a vector bundle with zero section $\iota: L \hookrightarrow P \backslash\{p\}$, i.e. $\xi_{p, L}$ is a tubular neighborhood of $L$ in $P$. Hence we get the following corollary.

(5.3) Corollary. For every line $L$ and every point $p$ not on $L$ the bundle $\xi_{p, L}$ is isomorphic to the normal bundle $\nu L$ of $L$ in $P$.

Together with Lemma (5.1) this corollary gives

(5.4) Corollary. Any two bundles $\xi_{p, L}$ and $\xi_{q, K}$ are isomorphic as smooth vector bundles.

Proof. According to Corollary (5.3) we have $\xi_{p, L} \cong \xi_{x, L}$ for any point $x \in P \backslash L$. Choosing $x \in P \backslash(K \cup L)$ we get $\xi_{p, L} \cong \xi_{x, L} \cong \xi_{x, K} \cong \xi_{q, K}$, where the isomorphism in the middle is due to Lemma (5.1).

Buchanan, Hähl, and Löwen ([12], (1.3)) proved that the point space $Q$ of a compact connected projective plane is $n$-homogeneous, i.e. for any two sets $A$ and $B$ of $n$ distinct points and for any bijection $\varphi: A \rightarrow B$ there is a homeomorphism $\Phi: Q \rightarrow Q$ with $\left.\Phi\right|_{A}=\varphi$. The next corollary is in the same spirit as this result.

(5.5) Corollary. For any two points $p, q \in P$ and any line $L \in \mathcal{L}$ not passing through one of the points $p$ and $q$ there is a homeomorphism $\Phi: P \rightarrow P$ such that $\Phi(K) \in \mathcal{L}_{q}$ for every line $K \in \mathcal{L}_{p}$. Moreover, the map $\Phi$ fixes every point on $L$ and is isotopic rel $L$ to the identity.

Proof. By Corollary (5.4) and (5.3) and the fact that any two tubular neighborhoods are isotopic, the total spaces of the bundles $\xi_{p, L}$ and $\xi_{q, L}$ are thus isotopic rel $L$. This isotopy can be extended to all of $P$ in order to give an isotopy $F: P \times[0,1] \rightarrow P$ of homeomorphisms $F_{t}$. Clearly, the homeomorphism $\Phi=F_{1}$ has the desired properties.

The dual of Lemma (5.1) showed that any two line pencils of $\mathcal{P}$ are embedded equivalently in $\mathcal{L}$. A slight modification of the proof of this lemma shows that this is also true for the subsets $\mathcal{F}_{p}:=\mathcal{L}_{p} \times\{p\}=\pi_{P}^{-1}(p)$ of the flag space $\mathcal{F}$. More precisely, we have

(5.6) Lemma. For any two points $p_{0}$ and $p_{1}$ of $\mathcal{P}$ there is a diffeotopy $F: \mathcal{F} \times[0,1] \rightarrow \mathcal{F}$ with $F_{0}=\mathrm{id} \mathcal{F}$ and $F_{1}\left(\mathcal{F}_{p_{0}}\right)=\mathcal{F}_{p_{1}}$.

Now choose two points $p$ and $q$ in $\mathcal{P}$ and fix some line $L$ with $p, q \notin L$. Since the affine point set $A:=P \backslash L$ is diffeomorphic to $\mathbb{R}^{n}$ (cp. [4], (4.4)), there is a smooth path $\gamma$ in $A$ connecting $p$ with $q$. Let $\omega$ denote the antipodal mapping of $L \approx \mathbb{S}_{l}$. It is not clear whether or not the point rows (or the line pencils) are diffeomorphic to standard spheres, although 
smooth $l$-spheres are always standard if $l=1,2,3,5,6$. It is an open problem whether there are exotic structures on the 4-sphere. In the case $l=8$, Kervaire and Milnor, [19], proved that there is exactly one exotic 8-sphere. Unfortunately, the antipodal map (as well as any other fixed-point free map on the 8-sphere) is not smooth on the exotic 8-sphere, see the book of López de Medrano, [26]. For the following construction, however, we shall need a fixed point free diffeomorphism $\alpha$ of the point row $L$. Thus we have to assume that $L$ is diffeomorphic to the standard sphere $\mathbb{S}_{l}$. For $x \in P$ put $\mathcal{U}_{x}:=\pi_{\mathcal{L}}^{-1}\left(\mathcal{L}_{x}\right)$. The mapping

$$
\varphi: \mathcal{U}_{p} \times[0,1] \rightarrow \mathcal{F}:(x, K, t) \mapsto\left(\left(x \vee(K \wedge L)^{\alpha}\right) \wedge((K \wedge L) \vee \gamma(t)),(K \wedge L) \vee \gamma(t)\right)
$$

is an isotopy of embeddings with $\varphi_{0}=\mathrm{id} \mathfrak{U}_{p}$ and $\varphi_{1}\left(\mathcal{U}_{p}\right)=\mathcal{U}_{q}$. According to Lemma (3.7) the sets $\mathcal{U}_{x}$ are submanifolds of $\mathcal{F}$ which are closed and thus they are compact. Hence the isotopy extension theorem (see [17], $\S 8,(1.3)$ ) yields the following proposition.

(5.7) Proposition. For any two points $p, q \in P$ and any line $L \in \mathcal{L}$ not passing through one of the points $p$ and $q$ there is a diffeotopy $\Phi$ rel $\pi_{\mathcal{L}}^{-1}(L)$ of $\mathcal{F}$ with $\Phi_{0}=\mathrm{id} \mathcal{F}$ and $\Phi_{1}\left(\mathcal{U}_{p}\right)=\mathcal{U}_{q}$.

Since pointed lines are diffeomorphic to $\mathbb{R}^{l}$, the set $\mathcal{U}_{p} \backslash\{(x, x \vee p) \mid x \in L\}$ can be regarded as a smooth $\mathbb{R}^{l}$-bundle $\xi_{p}=\left(\mathcal{U}_{p} \backslash\{(x, x \vee p) \mid x \in L\},\{p\} \times \mathcal{L}_{p},(x, K) \mapsto(p, K)\right)$. The map $\Phi_{1}$ is a diffeomorphism between the total spaces $\mathrm{E}\left(\xi_{p}\right)$ and $\mathrm{E}\left(\xi_{q}\right)$. Moreover, the map $\Phi_{1}$ maps fibres of $\xi_{p}$ onto fibres of $\xi_{q}$. However, the base space of $\xi_{p}$ is not mapped onto the base space of $\xi_{q}$. Nevertheless, the image $\Phi_{1}\left(\{p\} \times \mathcal{L}_{p}\right)$ is a smooth section of $\xi_{q}$ and hence is diffeotopic via some isotopy $\vartheta$ to the zero section $\iota:\{q\} \times \mathcal{L}_{q} \hookrightarrow \mathrm{E}\left(\xi_{q}\right)$. The composition of $\Phi_{1}$ with the diffeomorphism $\vartheta_{1}$ gives a diffeomorphism $\Psi$ between the total spaces of $\xi_{p}$ and $\xi_{q}$ which preserves the fibres and the zero sections. Reducing the structure group to $\mathrm{GL}_{l} \mathbb{R}$ we get

(5.8) Theorem. For any two points $p, q \in P$ and any line $L \in \mathcal{L}$ not passing through one of the points $p$ and $q$ the bundles $\xi_{p}$ and $\xi_{q}$ are isomorphic smooth vector bundles.

\section{References}

[1] André, J., Über nicht-desarguessche Ebenen mit transitiver Kollineationsgruppe, Math. Z. 60 (1954), 156-186

[2] Betten, D., 2-dimensionale differenzierbare projektive Ebenen, Arch. Math. 22 (1971), 304-309

[3] Bödi, R., Kramer, L., Differentiability of continuous homomorphisms between smooth loops, Results Math. 25 (1994), 13-19

[4] Bödi, R., Kramer, L., On homomorphisms of generalized polygons, Geom. Dedicata. 58 (1995), 1-14

[5] Bredon, G.E., Topology and Geometry, New York: Springer 1993

[6] Breitsprecher, S., Einzigkeit der reellen und der komplexen projektiven Ebene, Math. Z. 99 (1967), 429-432

[7] Breitsprecher, S., Projektive Ebenen, die Mannigfaltigkeiten sind, Math. Z. 121 (1971), 157-174 
[8] Breitsprecher, S., Zur topologischen Struktur zweidimensionaler projektiver Ebenen, Geom. Dedicata 1 (1972), 21-32

[9] Breuning, P., Translationsebenen und Vektorraumbündel, Mitt. Math. Sem. Gießen 86 (1970), 1-50

[10] Bröcker, T., Jänich, K., Einführung in die Differentialtopologie, Berlin: Springer 1973

[11] Bruck, R.H., Bose, R.C., The construction of translation planes from projective spaces, J. Algebra 1 (1964), 85-102

[12] Buchanan, T., Hähl, H., Löwen, R., Topologische Ovale, Geom. Dedicata 9 (1980), 401-424

[13] Buekenhout, F. (Ed.), Handbook of incidence geometry, Amsterdam: North Holland 1995

[14] Grundhöfer, T., Ternary fields of compact projective planes, Abh. Math. Sem. Univ. Hamburg 57 (1987), 87-101

[15] Grundhöfer, T., Hähl, H., Fibrations of spheres by great spheres over division algebras and their differentiability, J. Differential Geometry 31 (1990), 357-363

[16] Hähl, H., Differentiable fibrations of the (2n-1)-sphere by great $(n-1)-$ spheres and their coordinatization over quasifields, Results Math. 12 (1987), 99-118

[17] Hirsch, M.W., Differential Topology, New York: Springer 1976

[18] Husemoller, D., Fibre Bundles, New York: Springer 1975

[19] Kervaire, M.A, Milnor, J.W., Groups of homotopy spheres, Ann. Math. 77 (1963), 504-537

[20] Kirby, R.C., Siebenmann, L.C., Foundational essays on topological manifolds, smoothings, and triangulations, New Jersey: Princeton University Press 1977

[21] Kister, J., Microbundles are fibre bundles, Ann. Math. 80 (1964), 190-199

[22] Kosinski, A. A., Differential Manifolds, San Diego: Academic Press 1993

[23] Kuiper, N.M., Lashof, R.K., Microbundles and bundles, I., Invent. Math. 1 (1966), 1-17

[24] Kramer, L., The topology of smooth projective planes, Arch. Math. 63 (1994), 85-91

[25] Kramer, L., Holomorphic polygons, Math. Z. 223 (1996), 333-341

[26] López de Medrano, S., Involutions on Manifolds, Berlin-Heidelberg-New York: Springer 1971

[27] Löwe, H., Shear planes, Geom. Dedicata 52 (1994), 87-104

[28] Löwen, R., Vierdimensionale stabile Ebenen, Geom. Dedicata 5 (1976), 239-294

[29] Löwen, R., Symmetric planes, Pacific J. Math. 84 (1979), 367-390

[30] Löwen, R., Topology and dimension of stable planes : On a conjecture of $H$. Freudenthal, J. Reine Angew. Math. 343 (1983), 108-122

[31] Löwen, R., Compact spreads and compact translation planes over locally compact fields, J. Geom. 36 (1989), 110-116

[32] Milnor, J., Microbundles, Part I, Topology, Suppl. 13 (1964), 53-80

[33] Otte, J., Differenzierbare Ebenen, Dissertation Kiel 1992

[34] Otte, J., Smooth Projective Translation Planes, Geom. Dedicata 58 (1995), 203-212

[35] Salzmann, H., Topological Planes, Advances in Math. 2 (1967), 1-60

[36] Salzmann, H., Betten, D., Grundhöfer, T., Hähl, H., Löwen, R., Stroppel, M., Compact Projective Planes, De Gruyter 1995

[37] Siebenmann, L., Guillou, L., Hähl, H., Les voisinages ouverts réguliers, Ann. scient. Éc. Norm. Sup. 6 (1973), 253-293

[38] Stewart, T.E., On groups of diffeomorphisms, Proc. Amer. Math. Soc. 11 (1960), 559-563

[39] Stroppel, M., Achtdimensionale stabile Ebenen mit quasieinfacher Automorphismengruppe, Dissertation, Tübingen 1990

[40] Stroppel, M., Stable planes with large groups of automorphisms: the interplay of incidence, topology, and homogeneity, Habilitationsschrift, Darmstadt 1993

\author{
Richard Bödi \\ Mathematisches Institut \\ Universität Tübingen \\ Auf der Morgenstelle 10 \\ 72076 Tübingen \\ GERMANY
}

richard.boedi@uni-tuebingen.de
Eingegangen am 6. Januar 1997 